\title{
Rilonacept (Interleukin-1 Inhibition) for the Treatment of Pericarditis
}

\author{
Tom Kai Ming Wang ${ }^{1} \cdot$ Allan L. Klein ${ }^{1}$
}

Accepted: 23 September 2021 / Published online: 6 January 2022

( ) The Author(s), under exclusive licence to Springer Science+Business Media, LLC, part of Springer Nature 2022, corrected publication 2022

\begin{abstract}
Purpose of Review We reviewed the contemporary literature and clinical trials to discuss the applications of the interleukin-1 (IL-1) inhibitor rilonacept to treat pericarditis, with regards to pathophysiology, pharmacology, efficacy, and safety.

Recent Findings Rilonacept is an emerging novel agent for treating recurrent pericarditis, with phase II and III clinical trials recently published. Rilonacept rapidly resolved pericarditis pain and inflammation, markedly reduced recurrent pericarditis episodes, and had few adverse events indicating a high safety profile.

Summary Recurrent pericarditis is associated with significant morbidity and unmet need for novel therapies. Inflammasomes and the IL-1 pathways were found to be critical in its pathophysiology, leading to IL-1 inhibitors being developed. The high efficacy and safety of rilonacept for recurrent pericarditis means it could potentially be considered as a second-line therapy ahead of or as an alternative to corticosteroids, and highlight the great promise of targeted immunomodulatory therapy in this field.
\end{abstract}

Keywords Rilonacept $\cdot$ Interleukin-1 inhibitor $\cdot$ Pericarditis $\cdot$ Recurrent pericarditis $\cdot$ Inflammation

\section{Introduction}

Pericarditis is the commonest form of pericardial disease characterized by inflammation of the pericardial sac, with incidence of 27.7/100,000 per year [1]. The main classes of etiologies are idiopathic (80-90\%, presumed to be postviral), infective (including viral, bacterial, fungal, and parasitic), autoimmune, neoplastic, metabolic, iatrogenic (from cardiac surgery and intervention), or trauma and treatment-related (such as medications and radiation) [2, $3]$. Based on time course, pericarditis can be divided into acute $(<4-6$ weeks), incessant $(>4-6$ weeks but $<3$ months without remission), recurrent ( $2+$ episodes of acute pericarditis with symptom-free interval $>4-6$ weeks), and chronic

This article is part of the Topical Collection on New Therapies for Cardiovascular Disease

Allan L. Klein

kleina@ccf.org

Tom Kai Ming Wang

wangt2@ccf.org

1 Center for the Diagnosis and Treatment of Pericardial Diseases, Section of Cardiovascular Imaging, Heart, Vascular and Thoracic Institute, Cleveland Clinic, Cleveland, $\mathrm{OH}, \mathrm{USA}$ pericarditis (lasting $>3$ months) [2]. Diagnosis of pericarditis based on the European Society of Cardiology criteria includes at least 2 of pericarditic chest pain, pericardial rub on examination, new widespread ST elevation or PR depression on ECG, and new or worsening pericardial effusion, while supporting information include elevated inflammatory markers and imaging evidence of pericardial inflammation (such as edema and delayed gadolinium enhancement on magnetic resonance imaging) [2,4]. The standard first-line therapies for pericarditis are non-steroidal anti-inflammatories for 1-2 weeks and colchicine for 3 months, while corticosteroids may be used if intolerant or refractory to first-line therapies [2]. Unfortunately, a significant minority $15-30 \%$ of these patients develop recurrent pericarditis even with adequate first-line therapy during the initial episode, and is associated with morbidities, poor quality of life, and strain on healthcare resources $[2,5]$. Therefore, there is unmet need in the adequate treatment of recurrent pericarditis, including to spare the adverse effects of medium- to longterm NSAIDs and steroids. Apart from emerging therapies such as azathioprine and human immunoglobulins, recent advances and clinical trials in the field of targeted immunotherapy, especially interleukin-1 (IL-1) inhibitors like anakinra and rilonacept, have demonstrated efficacy and potential in managing this condition $[2,6,7,8 \bullet \bullet]$. This review 
focuses on discussing the pathophysiology, pharmacology, current evidence, and clinical applications of rilonacept to treat pericarditis.

\section{Pathophysiology}

Although not fully understood, the pathophysiology of pericarditis include several immune pathways as part of the body's innate and adaptive immunity systems that together lead to inflammation (Fig. 1) [9]. Broadly speaking, environmental irritants such as infectious, autoimmune, trauma, iatrogenic, and metabolic disturbances trigger off pericardial inflammation through the body's immune system that amplifies and sustains these processes in a genetically susceptible host [10]. Initially it was thought that inappropriate adaptive immunity played a major role in recurrent pericarditis, including molecular mimicry and cross-reactivity of foreign antigens along with T-cell activation due to superantigens from infections, along with frequent association with autoimmune diseases such as systemic lupus erythematosus and rheumatoid arthritis, and correlating with relapse events [11, 12]. Recent studies have shown innate immunity to be pivotal in the pathogenesis of idiopathic pericarditis, with common mechanisms to other autoinflammatory diseases such as tumor necrosis factor alpha-associated periodic syndrome and familial Mediterranean fever [9, 13]. Key clinical features of these conditions include fluctuant but abrupt fever episodes, polyserositis and arthralgias, markedly abnormal inflammatory markers, and quiescent intervals in between. These diseases typically demonstrate unprovoked multisystem inflammation from innate immunity disturbances without significant levels of autoantibodies or antigen-specific $\mathrm{T}$ cells [14]. The inflammasome and its release of interleukin-1 (IL-1) has a critical role in these conditions, distinguishing autoinflammatory diseases from autoimmune diseases.

The inflammasome is a cystolic macromolecule made up of a nucleotide-binding oligomerization domain-like

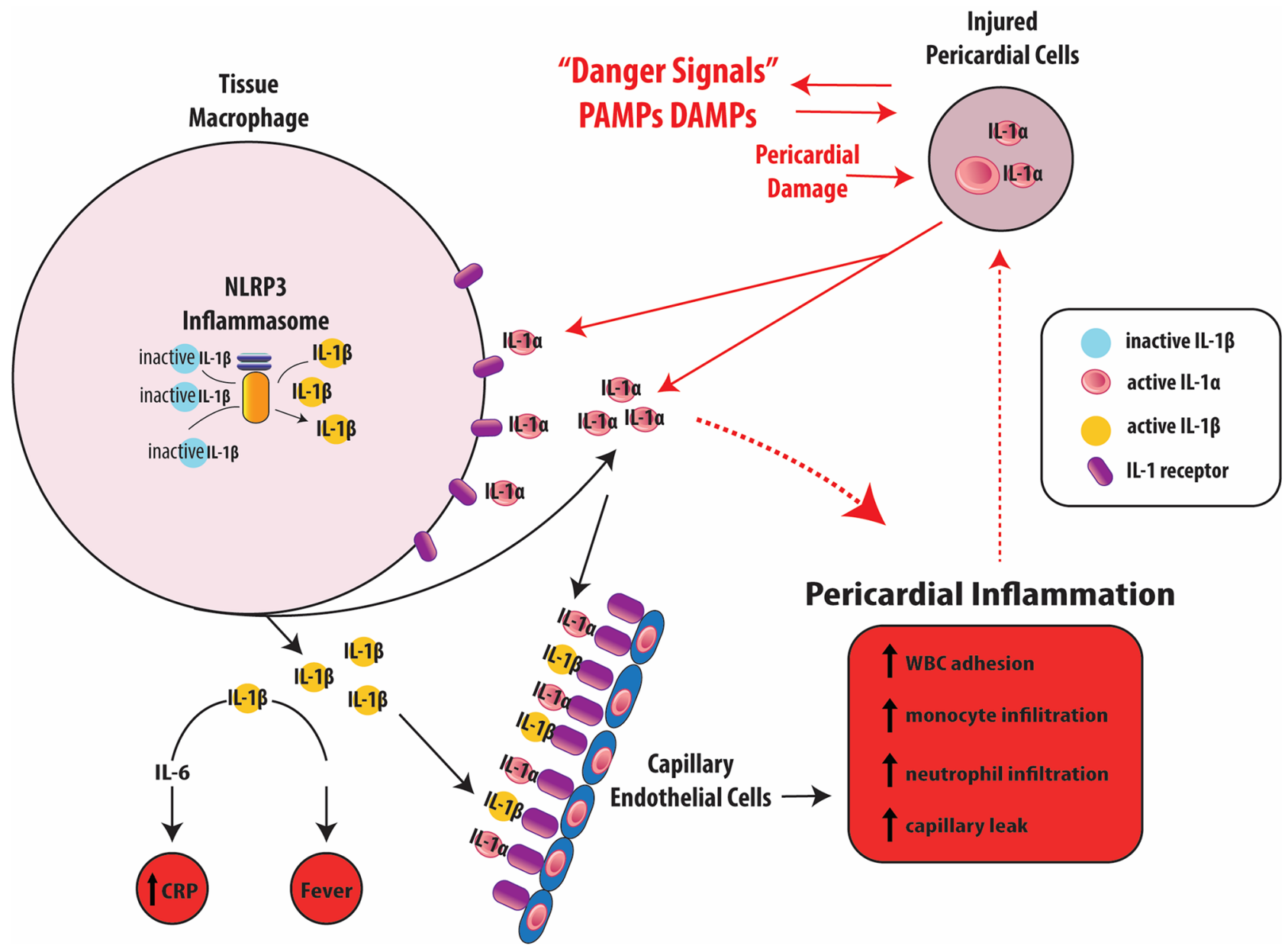

Fig. 1 Pathophysiological mechanisms of recurrent pericarditis (modified from RHAPSODY trial design study with permission from [22•]; copyright 2021. Elsevier Science \& Technology Journals) 
receptor (NLR) as a sensor (most commonly NLR pyrin domain-containing 3 or NLRP3), adaptor protein ASC, and procaspase-1 enzyme [15]. The NLRP3 sensor has a diverse range of activating triggers such as pathogen-associated molecular patterns, damage-associated molecular patterns, tumor necrosis factor alpha, viruses, monosodium urate crystals, and gain-of-function mutations. NLRP3 activation switches on caspase-1 which cleaves the pro-IL-1 made via NFKB transcription factor in the nucleus to IL-1 before releasing it outside the cell $[15,16]$. IL-1 primarily recruits myeloid lineage effector cells including neutrophils, monocytes, and macrophages to the site of injury and inflammation such as the pericardium. Given the critical role of the IL-1 pathway in autoinflammatory diseases like pericarditis, it has become a promising pharmacological target for drug development of IL-1 inhibitors including rilonacept.

\section{Interleukin-1 Inhibitors}

Before rilonacept was studied for recurrent pericarditis, there were other IL-1 inhibitors used in research and clinical practice, albeit restricted, as shown in Table 1 . The main agent is anakinra, which is FDA-approved for rheumatoid arthritis in patients who have failed at least 1 disease-modifying antirheumatic drug, cryopyrin-associated periodic syndromes, and interleukin-1 receptor antagonist deficiency [17]. It is a recombinant human IL-1Ra that inhibits the IL- $1 \alpha$ and IL-1 $\beta$. Anakinra was studied in the "Anakinra-Treatment of Recurrent Idiopathic Pericarditis (AIRTRIP)" multi-center double-blinded randomized trial at idiopathic multiple recurrent pericarditis patients with colchicine resistance, corticosteroid dependence, and elevated C-reactive protein [7]. All patients received $2 \mathrm{mg} / \mathrm{kg}$ (100 $\mathrm{mg}$ maximum) daily for 2 months, and in 21 patients with resolution of pericarditis were randomized to continuing anakinra or switching to placebo. Anakinra led to significant reduction in recurrence ( $2 / 11$ versus $9 / 10$ patients), with good safety profile (infrequent apart from local skin reaction). Similar results were reported from the International Registry of Anakinra for Pericarditis cohort including 224 recurrent pericarditis patients, whereby anakinra was associated with lower rates of pericarditis recurrence, hospital visit and hospitalization, and more patients coming off corticosteroids [6]. As a result, anakinra is typically a third-line therapeutic option who is resistant to other agents.

Another IL-1 inhibitor worthy of note is the selective monoclonal antibody against IL- $1 \beta$, canakinumab. Its current FDA indications are in several periodic fever syndromes (cryopyrin-associated periodic syndromes, tumor necrosis factor receptor-associated periodic syndrome, hyperimmunoglobulin D syndrome, and familial Mediterranean fever) and active systemic juvenile idiopathic arthritis [18]. In cardiology, canakinumab gained recent interest in the "Canakinumab Antiinflammatory Thrombosis Outcomes Study (CANTOS)" randomized trial of 10,061 patients with elevated C-reactive protein and history of myocardial infarction, where it significantly reduced the primary composite cardiovascular event endpoint by dampening inflammation [19]. The evidence for pericarditis is limited to case reports and series however, with mixed level of efficacy reported and some with treatment failure, and therefore are rarely used in clinical practice even compared with anakinra [20, 21].

Table 1 Characteristics of interleukin-1 inhibitors

\begin{tabular}{|c|c|c|c|}
\hline Drug & Anakinra & Canakinumab & Rilonacept \\
\hline Mechanism & $\begin{array}{l}\text { Recombinant human protein inhibiting } \\
\text { IL- } 1 \alpha \text { and IL-1 } \beta\end{array}$ & $\begin{array}{l}\text { Monoclonal IgG antibody inhibiting } \\
\text { IL-1 } \beta\end{array}$ & $\begin{array}{l}\text { Dimeric fusion protein inhibiting IL-1 } \alpha \\
\text { and IL- } 1 \beta\end{array}$ \\
\hline Administration & Subcutaneous, intravenous & Subcutaneous & Subcutaneous \\
\hline Dosing & $\begin{array}{l}\text { No loading dose } \\
2 \mathrm{mg} / \mathrm{kg} \text { (up to } 100 \mathrm{mg} \text { ) daily }\end{array}$ & $\begin{array}{l}\text { No loading dose } \\
4 \mathrm{mg} / \mathrm{kg} \text { or } 150 \mathrm{mg} \text { (single dose) every } \\
4-8 \text { weeks }\end{array}$ & $\begin{array}{l}\text { Loading } 320 \mathrm{mg}(4.4 \mathrm{mg} / \mathrm{kg} \text { up to } 320 \mathrm{mg} \\
\text { in } 12-17 \text { year olds) } \\
\text { Maintenance } 160 \mathrm{mg} \text { weekly }(2.2 \mathrm{mg} / \mathrm{kg} \\
\text { up to } 160 \mathrm{mg} \text { in } 12-17 \text { year olds })\end{array}$ \\
\hline Dose adjustment & $\begin{array}{l}\text { Renal: creatinine clearance }<30 \mathrm{~mL} / \\
\text { min consider alternate day dosing } \\
\text { Hepatic: none necessary }\end{array}$ & $\begin{array}{l}\text { Renal: none necessary } \\
\text { Hepatic: none necessary }\end{array}$ & $\begin{array}{l}\text { Renal: none necessary } \\
\text { Hepatic: none necessary }\end{array}$ \\
\hline Half-life & $4-6 \mathrm{~h}$ & 26 days & 7 days \\
\hline Adverse events & $\begin{array}{l}\text { Injection site reactions, infections, } \\
\text { hepatitis }\end{array}$ & $\begin{array}{l}\text { Injection site reactions, infections, } \\
\text { neutropenia }\end{array}$ & $\begin{array}{l}\text { Injection site reactions, infections, dys- } \\
\text { lipidemia, neutropenia }\end{array}$ \\
\hline Monitoring & $\begin{array}{l}\text { C-reactive protein, complete blood } \\
\text { count, infection, baseline tuberculosis, } \\
\text { and hepatitis screening }\end{array}$ & $\begin{array}{l}\text { C-reactive protein, complete blood } \\
\text { count, infection, baseline tuberculosis } \\
\text { screening }\end{array}$ & $\begin{array}{l}\text { C-reactive protein, complete blood count, } \\
\text { lipid profile, infection }\end{array}$ \\
\hline
\end{tabular}




\section{Pharmacological Properties of Rilonacept}

\section{Chemistry and Indications}

The key features of rilonacept are summarized in Table 1 . Rilonacept is a dimeric fusion protein (molecular weight $251 \mathrm{kDa}$ ) made up of an extracellular human IL-1 receptor component with ligand-binding domains, IL-1 receptor accessory protein, and $\mathrm{Fc}$ portion of human immunoglobulin G1 [22•, 23]. It works as a soluble decoy receptor binding to IL-1 alpha or beta with higher affinity than cell surface receptors to preventing receptor binding and therefore the downstream inflammatory response. Pharmacokinetic data suggest half-life of approximately 26 days and 6 weeks to reach steady state, with similar concentrations in men and women, and therapeutic index is high. The Food and Drug Administration (FDA) authorized rilonacept to be used in three types of conditions: initially in 2008 for (a) familial cold autoinflammatory syndrome, cryopyrin-associated periodic syndromes, and Muckle-Wells syndrome; (b) IL-1 receptor antagonist deficiency; and most recently in March 2021 (c) recurrent pericarditis, which will be discussed further [23].

\section{Dosing and Administration}

Rilonacept is administered subcutaneously as an injection [23]. In adults at least 18 years of age, the standard dosing is initial loading of $320 \mathrm{mg}$, followed by once-weekly $160-\mathrm{mg}$ injections. In pediatric patients 12-17 years of age, loading dose is $4.4 \mathrm{mg} / \mathrm{kg}$ up to $320 \mathrm{mg}$, then weekly injections $2.2 \mathrm{mg} / \mathrm{kg}$ up to $160 \mathrm{mg}$ thereafter. It should not be administered more than once a week. No dosing adjustment is advised for patients with renal or hepatic impairment or geriatric patients. The treatment course length has not been defined and warrants further study. Rilonacept can be stored in the refrigerator at $2-8{ }^{\circ} \mathrm{C}$, away from light, and using aseptic technique, mixing the drug from vial with sterile water and inject within $3 \mathrm{~h}$ of mixing. Alternate injection sites between upper arms, abdomen, and thigh and avoid red, tender, or bruised sites.

\section{Adverse Events and Precautions}

The commonest side effects of rilonacept are injectionsite reactions and upper respiratory tract infections, while other reported adverse events include increased risk in serious infection, antibody development, hypoesthesia, cough, sinusitis, diarrhea, hypersensitivity reactions, and derangement of non-fasting lipid profile including increased
Fig. 2 Kaplan-Meier survival curve results of the RHAPSODY phase III trial $\left[8^{\bullet \bullet}\right.$ ] A pain score and C-reactive protein levels over time and $\mathbf{B}$ freedom from recurrent pericarditis events (reprinted

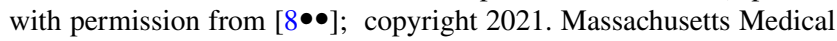
Society)

high- and low-density lipoprotein cholesterol and triglyc-

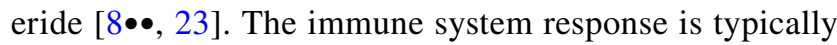
dampened with IL-1 blockade; therefore, rilonacept should not be administered with other agents that inhibit IL-1, such as tumor necrosis factor inhibitors to avoid serious infections [23]. Caution should be applied when adding rilonacept to other immunosuppressive agents. There are theoretical concerns for impaired response against malignancies which have not been thoroughly studied. Blood tests to monitor while on rilonacept considering adverse events include complete blood count with differential, lipid profile, and for efficacy C-reactive protein.

\section{Specific Scenario Considerations}

Several clinical settings are worth mentioning with regards to administering rilonacept. There are no human controlled studies to date in pregnant women evaluating effects on the fetus and mother, although animal studies have shown a slight increase in risk of stillbirth and lumbar ribs and the drug is placed under category $\mathrm{C}$, meaning to only use when benefits outweigh risk; otherwise, it should be discontinued before pregnancy [23]. There is also lack of data pertaining to rilonacept use during breastfeeding so caution is warranted. In pediatric populations, a small number of adolescent patients 12 years and above were part of the rilonacept pericarditis phase II and III studies discussed

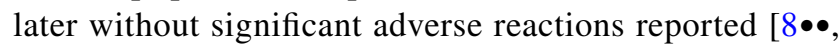
$24 \bullet$. As a result, rilonacept can be used in these patients, and rilonacept and anakinra have also been used in this age group for treating rare genetic disorders such as familial cold autoinflammatory syndrome and Mucky-Wells syndrome, while rilonacept is untested in those who are even younger [25, 26]. In terms of immunizations, IL-1 blockade have theoretical effects of impairing the immune system's response, so ideally, required vaccines are administered before rilonacept is started. Coronavirus-19 (COVID-19) vaccine studies did not include patients on IL-1 inhibitors to test whether vaccine efficacy is affected by these agents [27]. Although rilonacept has not yet been specifically assessed in COVID-19 infection trials, other IL-1 inhibitors such as canakinumab have been studied as a therapeutic agent for this condition, and early results found signals towards efficacy with no significant adverse events, and so extrapolating these results, rilonacept could probably be cautiously used in this setting [28]. 
A)

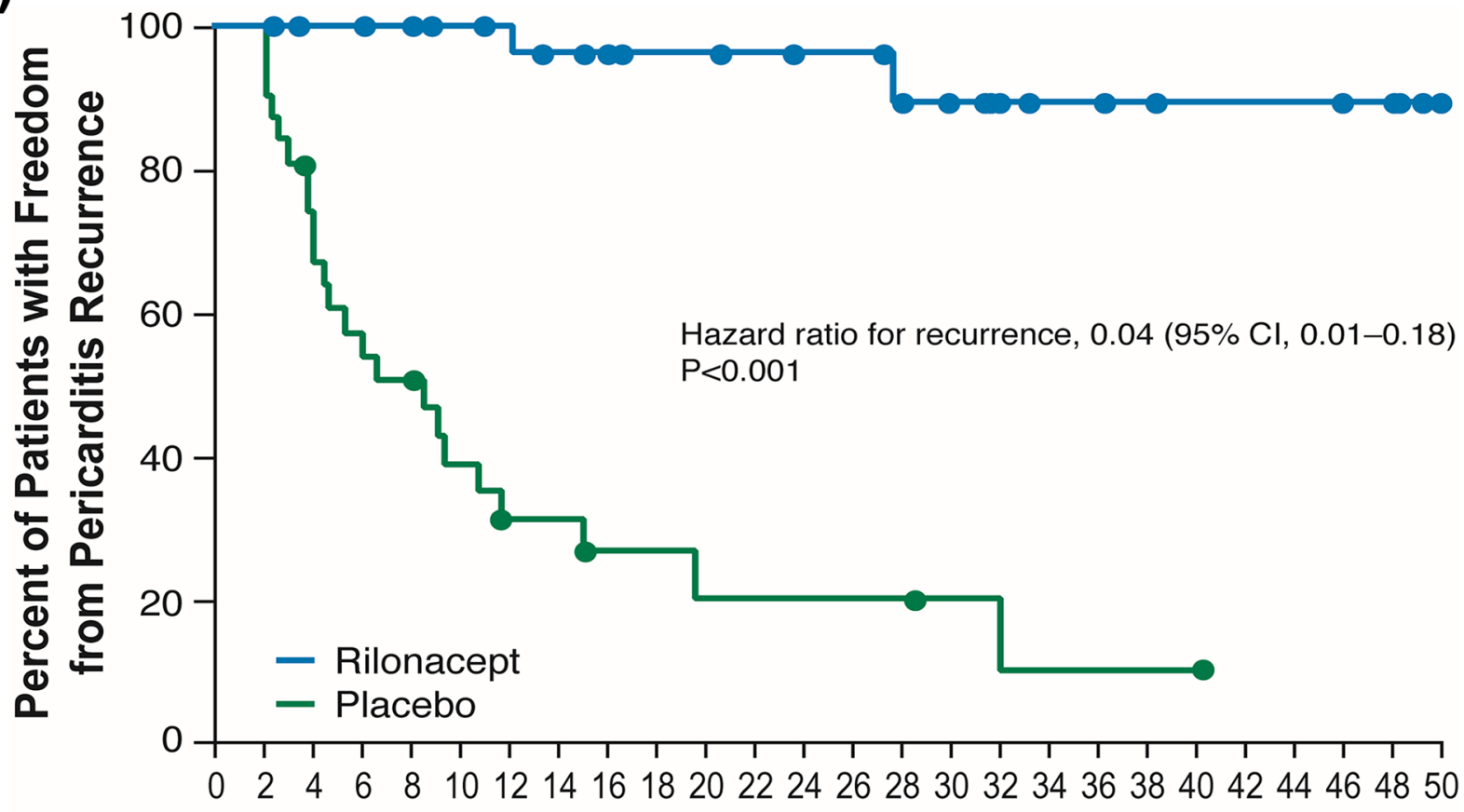
Weeks

No. of Patients $\begin{array}{llllllllllllllllllllllllllllll}\text { Rilonacept } & 30 & 30 & 28 & 27 & 26 & 24 & 23 & 21 & 20 & 17 & 17 & 16 & 15 & 15 & 11 & 11 & 9 & 7 & 7 & 6 & 5 & 5 & 5 & 5 & 4 & 1 \\ \text { Placebo } & 31 & 31 & 22 & 17 & 15 & 10 & 7 & 7 & 4 & 4 & 3 & 3 & 3 & 3 & 2 & 2 & 2 & 1 & 1 & 1 & 1 & & & & & \end{array}$

B)

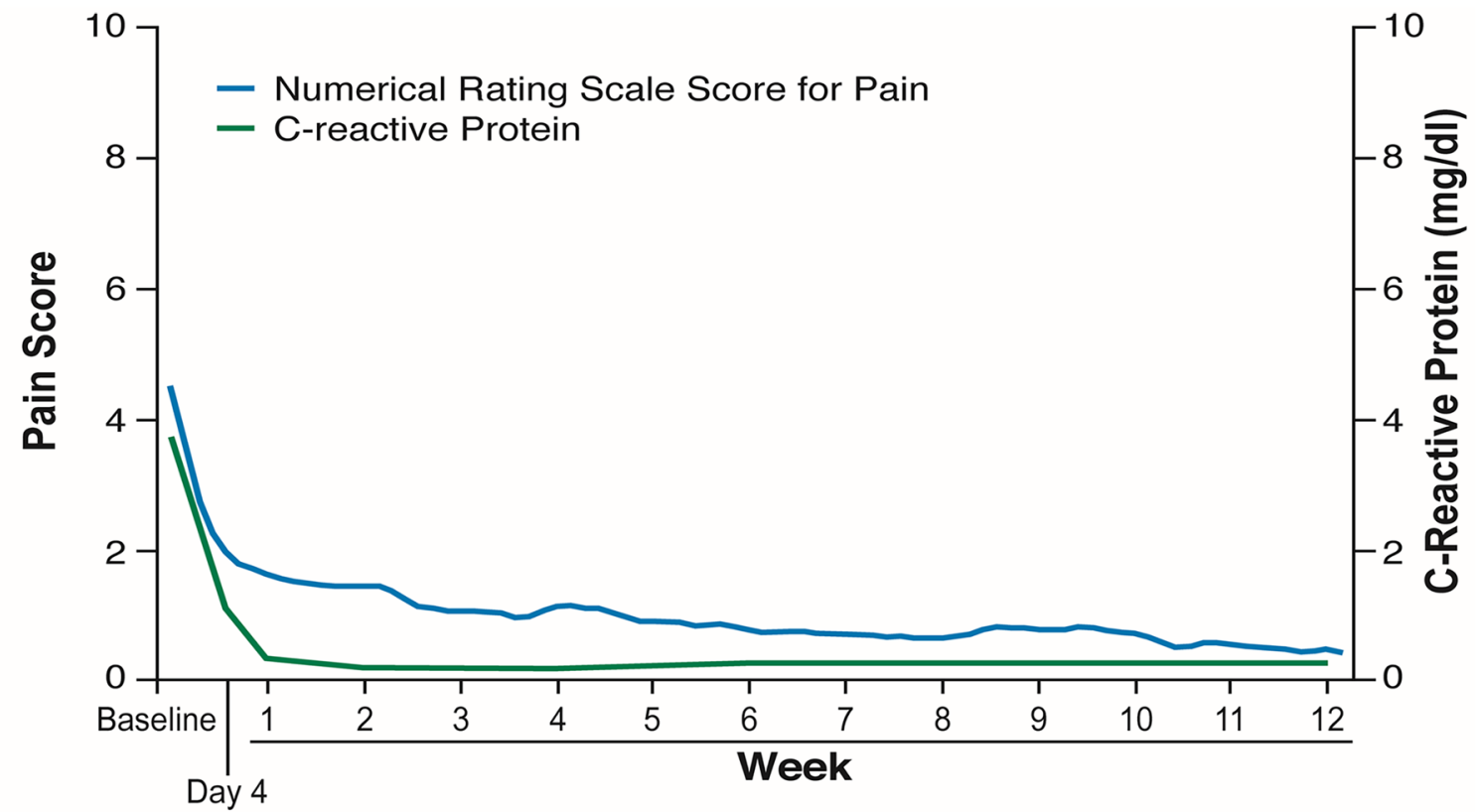

No. of Patients

Pain Score

No. of Patients

Mean Value

C-Reactive Protein

No. of Patients

$86 \quad 86 \quad 85$

Hazard ratio for recurrence, $0.04(95 \% \mathrm{Cl}, 0.01-0.18)$

$\mathrm{P}<0.001$

Mean Value

84
4.50

$\begin{array}{cc}84 & 8 \\ 1.60 & 1.43\end{array}$

$\begin{array}{lll}85 & 79 & 82\end{array}$

$\begin{array}{lll}3.70 & 1.000 .30 & 0.15\end{array}$

$\begin{array}{cc}85 & 85 \\ 83 & 83 \\ 1.04 & 1.13 \\ & 79 \\ & 0.13\end{array}$

$85 \quad 85$

$\begin{array}{cc}84 & 83 \\ 0.86 & 0.78\end{array}$

$84 \quad 84$

84

83

82

$\begin{array}{cc}83 & 82 \\ 0.68 & 0.61\end{array}$

81
0.76

82
0.69

0.53

79 


\section{Clinical Data and Applications of Rilonacept in Pericarditis}

\section{Phase II Data}

The clinical utility of rilonacept was rigorously tested in a series of clinical trials. The main phase II clinical trial was a multicenter open-label single-arm study of 25 adult patients with recurrent pericarditis from idiopathic or postpericardiotomy etiologies and either symptomatic or steroid dependent with at least 2 recurrences previously (confirmed with C-reactive protein $>1 \mathrm{mg} / \mathrm{dL}$ or delayed gadolinium enhancement on magnetic resonance imaging), published in 2020 [24•]. Rilonacept was administered subcutaneously with a 320-mg loading and 160-mg weekly maintenance for 6 weeks total, followed by optional extension period on treatment for 18 weeks. They found decreases in the pericarditis pain numeric rating scale (4.5 to 0.7$)$, C-reactive protein $(4.62$ to $0.38 \mathrm{mg} / \mathrm{dL}$, with 9 days of median time to normalization), and incidence of recurrent pericarditis from 3.9 to 0.18 episodes per year, and 11 of 13 patients on steroids at baseline had steroid discontinuation and 2 had steroid tapering. The majority $92 \%$ of adverse events were graded as mild or moderate, including $60 \%$ with injection site reactions, $16 \%$ with nasopharyngitis, $12 \%$ with arthralgia, and $12 \%$ with diarrhea, while only 1 serious adverse event occurred leading to rilonacept discontinuation. Increase in non-fasting cholesterol and triglycerides were noted, with no data on cardiovascular outcomes. It was therefore concluded that rilonacept was associated with rapid and sustained pain resolution, inflammatory marker normalization, and quality of life improvement with low adverse event rates.

\section{Phase III Trial}

The landmark phase III study is "Rilonacept Inhibition of Interleukin-1 Alpha and Beta for Recurrent Pericarditis: a Pivotal Symptomatology and Outcomes Study (RHAPSODY)," a multicenter, event-driven, randomized, parallel, doubleblinded, and placebo-controlled trial published in 2021, assessing the efficacy and safety of rilonacept in 86 patients with recurrent pericarditis $[8 \bullet \bullet]$. Patients were eligible with acute symptoms of recurrent pericarditis $(2+$ prior episodes $)$ and elevated systemic inflammatory markers (C-reactive protein $\geq 1 \mathrm{mg} / \mathrm{dL}$ ), and were characterized by mean age of 44.7 years, $57 \%$ women, $93 \%$ white race, idiopathic $85 \%$ and post-pericardiotomy syndrome $14 \%$, mean of 4.7 prior pericarditis episodes and 2.4 years since diagnosis, baseline medications colchicine $80 \%$, non-steroidal anti-inflammatory $67 \%$ and steroid $49 \%$, pain score 6.2 , and C-reactive protein $6.2 \mathrm{mg} / \mathrm{dL}$. There is a 12-week run-in-period for all patients to commence rilonacept and discontinue background medications, and then patients were randomized to weekly rilonacept monotherapy continuation or placebo. The primary efficacy endpoint was time-to-first pericarditis recurrence at 16-week follow-up, and other efficacy and safety endpoints were evaluated. Rilonacept led to a median normalization in symptoms of 5 days and C-reactive protein in 7 days, and significantly reduced pericarditis recurrence events (overall $6.7 \%$ versus $74.2 \%$, hazard ratio 0.04 ), along with having persistent clinical response ( $81 \%$ versus $20 \%$ at 16 weeks), and increased the days with no or minimal pain (Fig. 2). The most frequent adverse event was injection-site reactions (34\%) and upper respiratory tract infection (23\%), and there were 4 patient adverse events resulting in rilonacept discontinuation from extrinsic allergic alveolitis, alopecia, erythema, and systemic

Table 2 Proposed updated treatment algorithm for recurrent pericarditis including rilonacept

\begin{tabular}{ll}
\hline Pericarditis event & Treatment \\
\hline Acute pericarditis & - NSAIDs (week(s)) \\
Recurrent pericarditis — first event & - Colchicine (3 months) \\
& - NSAIDs (weeks to months) \\
Recurrent pericarditis — second event or beyond & - Colchicine ( $\geq 6$ months) \\
& - NSAIDs (weeks to months) \\
& - Colchicine ( $\geq 6$ months) \\
Recurrent pericarditis — colchicine resistance and/or corti- & - Rilonacept can be considered, including ahead of corticosteroids, and other drugs \\
costeroid dependent & - NSAIDs \\
& - Colchicine \\
Recurrent pericarditis - rilonacept failure & - Rilonacept, including while tapering down other agents especially corticosteroids \\
& - Consider alternative interleukin-1 inhibitor (such as anakinra) \\
& - Intensify anti-inflammatory combinations \\
& - Pericardiectomy may be considered (last line) \\
\hline
\end{tabular}


allergic reaction, all during the run-in period. The authors concluded that rilonacept rapidly resolves recurrent pericarditis pain and inflammation with lower recurrent risk than placebo and adequate safety profile. Longer term follow-up is awaited to 24 months and beyond.

\section{Clinical Implications}

RHAPSODY was a positive trial highlighting the efficacy of rilonacept in treating recurrent pericarditis, that may be otherwise medically refractory, and is far superior to placebo with rapid symptomatic and biomarker response. Notable limitations of the RHAPSODY trial include modest sample size and power, limited follow-up duration to date, enrolling recurrent pericarditis patients only, and questions about the higher than expected primary endpoint rates in the placebo arm which may accentuate the reported efficacy of rilonacept $[2,29,30]$. Taking these into account, FDA approved rilonacept to treat recurrent pericarditis in March 2021 [23]. Rilonacept should be strongly considered as a second-line therapy either as an alternative to or even ahead of corticosteroids, given the adverse event profile of the latter. We therefore propose the treatment algorithm listed in Table 2. Further research and real-world experiences of rilonacept in pericarditis are warranted for ongoing review of its efficacy, safety monitoring, and considerations to expand indications.

\section{Conclusions}

In summary, to address the unmet clinical need to adequately treat recurrent pericarditis with its associated morbidity burden, there has been a recent blossoming of novel targeted immunomodulating therapies. Innate immunity including inflammasomes and IL-1 is increasingly understood as an important pillar in the pathophysiology of pericarditis and target for IL-inhibitors, with rilonacept at the forefront. Rilonacept has been shown in phase II and most recently the phase III RHAPSODY trial to be effective in rapidly suppressing pericarditis symptoms and inflammation, with significantly lower risk of recurrence and good safety profile. This was a landmark trial in the treatment for recurrent pericarditis, and rilonacept can be considered ahead of or as an alternative to corticosteroids in this condition. Further follow-up and studies are required to assess the medium- to long-term treatment course and clinical outcomes, applications in other forms of pericardial diseases, and identify other suitable targets to develop novel therapies.

\section{Compliance with Ethical Standards}

Conflict of Interest The authors declare that they have no conflict of interest.
Human and Animal Rights and Informed Consent This article does not contain any studies with human or animal subjects performed by any of the authors.

\section{References}

Papers of particular interest, published recently, have been highlighted as:

- Of importance

• Of major importance

1. Imazio M, Cecchi E, Demichelis B, Chinaglia A, Ierna S, Demarie D, et al. Myopericarditis versus viral or idiopathic acute pericarditis. Heart. 2008;94(4):498-501.

2. Adler Y, Charron P, Imazio M, Badano L, Baron-Esquivias G, Bogaert J, et al. 2015 ESC guidelines for the diagnosis and management of pericardial diseases: the Task Force for the Diagnosis and Management of Pericardial Diseases of the European Society of Cardiology (ESC) endorsed by: The European Association for Cardio-Thoracic Surgery (EACTS). Eur Heart J. 2015;36(42):2921-64.

3. Imazio M, Gaita F, LeWinter M. Evaluation and treatment of pericarditis: a systematic review. JAMA. 2015;314(14):1498-506.

4. Klein AL, Abbara S, Agler DA, Appleton CP, Asher CR, Hoit B, et al. American Society of Echocardiography clinical recommendations for multimodality cardiovascular imaging of patients with pericardial disease: endorsed by the Society for Cardiovascular Magnetic Resonance and Society of Cardiovascular Computed Tomography. J Am Soc Echocardiogr. 2013;26(9):9651012.e15.

5. Imazio M, Brucato A, Cemin R, Ferrua S, Maggiolini S, Beqaraj $\mathrm{F}$, et al. A randomized trial of colchicine for acute pericarditis. N Engl J Med. 2013;369(16):1522-8.

6. Imazio M, Andreis A, De Ferrari GM, Cremer PC, Mardigyan $\mathrm{V}$, Maestroni S, et al. Anakinra for corticosteroid-dependent and colchicine-resistant pericarditis: the IRAP (International Registry of Anakinra for Pericarditis) study. Eur J Prev Cardiol. 2019;27(9):956-64.

7. Brucato A, Imazio M, Gattorno M, Lazaros G, Maestroni S, Carraro M, et al. Effect of anakinra on recurrent pericarditis among patients with colchicine resistance and corticosteroid dependence: the AIRTRIP randomized clinical trial. JAMA. 2016;316(18):1906-12.

8.• Klein AL, Imazio M, Cremer P, Brucato A, Abbate A, Fang F, et al. Phase 3 trial of interleukin-1 trap rilonacept in recurrent pericarditis. N Engl J Med. 2021;384(1):31-41. Pivotal phase III randomized trial showing rilonacept's efficacy and safety including rapid resolution of pericarditis episodes and reduction in recurrent pericarditis events over placebo in recurrent pericarditis.

9. Gaspari S, Marsili M, Imazio M, Brucato A, Di Blasi Lo Cuccio $\mathrm{C}$, Chiarelli F, et al. New insights in the pathogenesis and therapy of idiopathic recurrent pericarditis in children. Clin Exp Rheumatol. 2013;31(5):788-94.

10. Imazio M. Idiopathic recurrent pericarditis as an immunemediated disease: current insights into pathogenesis and emerging treatment options. Expert Rev Clin Immunol. 2014;10(11):1487-92.

11. Brucato A, Imazio M, Cremer PC, Adler Y, Maisch B, Lazaros G, et al. Recurrent pericarditis: still idiopathic? The pros and cons of a well-honoured term. Intern Emerg Med. 2018. 
12. Cusick MF, Libbey JE, Fujinami RS. Molecular mimicry as a mechanism of autoimmune disease. Clin Rev Allergy Immunol. 2012;42(1):102-11.

13. Cantarini L, Lucherini OM, Cimaz R, Baldari CT, Bellisai F, Rossi Paccani $\mathrm{S}$, et al. Idiopathic recurrent pericarditis refractory to colchicine treatment can reveal tumor necrosis factor receptorassociated periodic syndrome. Int J Immunopathol Pharmacol. 2009;22(4):1051-8.

14. van Kempen TS, Wenink MH, Leijten EF, Radstake TR, Boes M. Perception of self: distinguishing autoimmunity from autoinflammation. Nat Rev Rheumatol. 2015;11(8):483-92.

15. Cremer PC, Kumar A, Kontzias A, Tan CD, Rodriguez ER, Imazio $\mathrm{M}$, et al. Complicated pericarditis: understanding risk factors and pathophysiology to inform imaging and treatment. $\mathrm{J}$ Am Coll Cardiol. 2016;68(21):2311-28.

16. Brucato A, Emmi G, Cantarini L, Di Lenarda A, Gattorno M, Lopalco G, et al. Management of idiopathic recurrent pericarditis in adults and in children: a role for IL-1 receptor antagonism. Intern Emerg Med. 2018;13(4):475-89.

17. KINERET [Anakinra]. U.S. Food and Drug Administration website. https://www.accessdata.fda.gov/drugsatfda_docs/label/ 2020/103950s51891bl.pdf. Revised December 2020. Accessed Jul 2021.

18. ILARIS [Canakinumab]. U.S. Food and Drug Administration website. https://www.accessdata.fda.gov/drugsatfda_docs/label/ 2020/125319s1001bl.pdf. Revised September 2020. Accessed Jul 2021.

19. Ridker PM, Everett BM, Thuren T, MacFadyen JG, Chang WH, Ballantyne C, et al. Antiinflammatory therapy with canakinumab for atherosclerotic disease. N Engl J Med. 2017;377(12):1119-31.

20. Kougkas N, Fanouriakis A, Papalopoulos I, Bertsias G, Avgoustidis $\mathrm{N}$, Repa A, et al. Canakinumab for recurrent rheumatic disease associated-pericarditis: a case series with long-term follow-up. Rheumatology (Oxford). 2018;57(8):1494-5.

21. Signa S, D’Alessandro M, Consolini R, Miniaci A, Bustaffa M, Longo C, et al. Failure of anti interleukin-1 beta monoclonal antibody in the treatment of recurrent pericarditis in two children. Pediatr Rheumatol Online J. 2020;18(1):51.

22.• Klein AL, Imazio M, Brucato A, Cremer P, LeWinter M, Abbate A, et al. RHAPSODY: Rationale for and design of a pivotal Phase 3 trial to assess efficacy and safety of rilonacept, an interleukin- $1 \alpha$ and interleukin- $1 \beta$ trap, in patients with recurrent pericarditis. Am Heart J. 2020;228:81-90. Pivotal phase III randomized trial study design and methodology for rilonacept versus placebo in recurrent pericarditis.

23. ARCALYST [Rilonacept]. U.S. Food and Drug Administration website. https://www.accessdata.fda.gov/drugsatfda_docs/label/ 2021/125249s049lbl.pdf. Revised March 2021. Accessed Jul 2021.

24. Klein AL, Lin D, Cremer PC, Nasir S, Luis SA, Abbate A, et al. Efficacy and safety of rilonacept for recurrent pericarditis: results from a phase II clinical trial. Heart. 2020;107(6):488-96.Initial phase II study experience for rilonacept found to lead to rapid and sustained improvement in pain and inflammatory markers in recurrent pericarditis patients with good safety.

25. Hoffman HM, Throne ML, Amar NJ, Cartwright RC, Kivitz AJ, Soo Y, et al. Long-term efficacy and safety profile of rilonacept in the treatment of cryopryin-associated periodic syndromes: results of a 72-week open-label extension study. Clin Ther. 2012;34(10):2091-103.

26. Picco P, Brisca G, Traverso F, Loy A, Gattorno M, Martini A. Successful treatment of idiopathic recurrent pericarditis in children with interleukin-1beta receptor antagonist (anakinra): an unrecognized autoinflammatory disease? Arthritis Rheum. 2009;60(1):264-8.

27. Baden LR, El Sahly HM, Essink B, Kotloff K, Frey S, Novak $\mathrm{R}$, et al. Efficacy and safety of the mRNA-1273 SARS-CoV-2 vaccine. N Engl J Med. 2021;384(5):403-16.

28. Sheng CC, Sahoo D, Dugar S, Prada RA, Wang TKM, Abou Hassan OK, et al. Canakinumab to reduce deterioration of cardiac and respiratory function in SARS-CoV-2 associated myocardial injury with heightened inflammation (canakinumab in Covid-19 cardiac injury: The three $\mathrm{C}$ study). Clin Cardiol. 2020;43(10):1055-63.

29. Imazio M, Belli R, Brucato A, Cemin R, Ferrua S, Beqaraj F, et al. Efficacy and safety of colchicine for treatment of multiple recurrences of pericarditis (CORP-2): a multicentre, doubleblind, placebo-controlled, randomised trial. Lancet (London, England). 2014;383(9936):2232-7.

30. Imazio M, Bobbio M, Cecchi E, Demarie D, Demichelis B, Pomari F, et al. Colchicine in addition to conventional therapy for acute pericarditis: results of the COlchicine for acute PEricarditis (COPE) trial. Circulation. 2005;112(13):2012-6.

Publisher's Note Springer Nature remains neutral with regard to jurisdictional claims in published maps and institutional affiliations. 